\title{
Next-Generation Immune Repertoire Sequencing as a Clue to Elucidate the Landscape of Immune Modulation by Host-Gut Microbiome Interactions
}

\author{
Tatsuo Ichinohe ${ }^{1 * t}$, Takahiko Miyama ${ }^{1 \dagger}$, Takakazu Kawase', Yasuko Honjo', \\ Kazutaka Kitaura ${ }^{2}$, Hiroyuki Sato ${ }^{2}$, Tadasu Shin-I3 and Ryuji Suzuki ${ }^{2,4}$
}

'Department of Hematology and Oncology, Research Institute for Radiation Biology and Medicine (RIRBM), Hiroshima University, Hiroshima, Japan, ${ }^{2}$ Repertoire Genesis Incorporation, Ibaraki, Japan, ${ }^{3}$ BITS Co. Ltd., Tokyo, Japan, ${ }^{4}$ Department of Rheumatology and Clinical Immunology, Clinical Research Center for Rheumatology and Allergy, National Hospital Organization Sagamihara Hospital, Sagamihara, Japan

\section{OPEN ACCESS}

Edited by:

Nadiya V. Boyko,

Uzhhorod National University, Ukraine

Reviewed by:

Pinyi Lu,

Biotherapeutics, Inc., United States Yanfei Zhang,

Geisinger Health System, United States

*Correspondence: Tatsuo Ichinohe tatsuo.ichinohe@gmail.com

tThese authors have contributed equally to this work.

Specialty section:

This article was submitted to

Nutritional Immunology,

a section of the journal

Frontiers in Immunology

Received: 10 December 2017

Accepted: 19 March 2018

Published: 03 April 2018

Citation:

Ichinohe T, Miyama T, Kawase T, Honjo Y, Kitaura K, Sato H, Shin-I T and Suzuki R (2018) Next-Generation Immune Repertoire Sequencing as a

Clue to Elucidate the Landscape of

Immune Modulation by Host-Gut

Microbiome Interactions.

Front. Immunol. 9:668.

doi: 10.3389/fimmu.2018.00668
The human immune system is a fine network consisted of the innumerable numbers of functional cells that balance the immunity and tolerance against various endogenous and environmental challenges. Although advances in modern immunology have revealed a role of many unique immune cell subsets, technologies that enable us to capture the whole landscape of immune responses against specific antigens have been not available to date. Acquired immunity against various microorganisms including host microbiome is principally founded on $T$ cell and B cell populations, each of which expresses antigen-specific receptors that define a unique clonotype. Over the past several years, high-throughput next-generation sequencing has been developed as a powerful tool to profile T- and B-cell receptor repertoires in a given individual at the single-cell level. Sophisticated immuno-bioinformatic analyses by use of this innovative methodology have been already implemented in clinical development of antibody engineering, vaccine design, and cellular immunotherapy. In this article, we aim to discuss the possible application of high-throughput immune receptor sequencing in the field of nutritional and intestinal immunology. Although there are still unsolved caveats, this emerging technology combined with single-cell transcriptomics/proteomics provides a critical tool to unveil the previously unrecognized principle of host-microbiome immune homeostasis. Accumulation of such knowledge will lead to the development of effective ways for personalized immune modulation through deeper understanding of the mechanisms by which the intestinal environment affects our immune ecosystem.

\footnotetext{
Keywords: next-generation immune repertoire sequencing, B-cell receptors, T-cell receptors, single-cell
} transcriptomics, human microbiome

\section{INTRODUCTION}

The jawed vertebrates have evolutionally acquired a unique immune system consisted of effector and regulator cells that can effectively respond or establish tolerance to millions of endoneous and environmental antigens in an epitope-specific manner (1). In this paradigm of the "adaptive" immune system, it is firmly believed that pre-existing repertoire of two types of lymphohematopoietic cells, 
classic T cells and B cells, predominantly determine the mode and pattern of immune responses in a given individual. At the single-cell level, these cells express a single type of unique antigen-specific receptors: T-cell receptor (TCR) for T cells and $\mathrm{B}$-cell receptor/immunoglobulin (BCR/Ig) for B cells that define a phenotypic clone or a clonotype of these immune cells. At the individual level, the estimated number of TCR and BCR/Ig clonotypes is from a few thousands to more than billions depending on the species of animal, which is believed to form the basis of the host ability to cope with innumerable immunologic threats. It is well-characterized that such huge diversity of antigen-specific receptors is created through somatic rearrangement of variable $(\mathrm{V})$, diversity (D), and joining (J) (or V and J) gene segments located in TCR- or BCR/Ig-encoding loci and the concomitant incorporation of random nucleotide insertions and deletions. However, the underlying mechanism by which these repertoires are ontogenetically developed and shaped is largely unknown. Furthermore, until recently, there had been no available technologies that comprehensively identify each of TCR and BCR/ Ig clonotypes constituting the whole adaptive immune cell repertoire.

During the past few decades, accumulative lines of evidence have indicated that the host microbiome and nutrients not only play key roles for balancing the host immunity in health and disease but have a tremendous influence on the generation and shaping of immune cell repertoire $(2,3)$. For instance, several groups have reported pivotal observations that alloimmune-mediated graft-versus-host reactions in the setting of hematopoietic cell transplantation and immune responses against malignant neoplasms triggered by immune checkpoint inhibition are associated with the abundance of distinct members of intestinal commensal flora (4-8). In this article, we highlight the recent advancement in high-throughput immune repertoire analysis by next-generation sequencing (NGS) and its possible application in future studies to elucidate previously unrecognized mechanisms of immune modulation by gut microbiota and oral nutrition.

\section{ROLES OF GUT MICROBIOME IN SYSTEMIC HOMEOSTASIS OF ADAPTIVE IMMUNE REPERTOIRE}

\section{Gastrointestinal Tract as a Key Site for Systemic Immune Modulation}

The gastrointestinal tract-associated lymphoid tissue is the largest immune compartment in the body. Therefore, it is quite reasonable to assume that the gut microbiome has a strong influence on the development and homeostasis of adaptive immune repertoire. In fact, the intestinal epithelium is an important anatomical site for the active interaction of the gut microbiome and various immune cells including antigen-presenting dendritic cells $(2,3,9)$. For instance, the induction of gut-resident Foxp3+ regulatory T cells (Tregs), a key modulator of immune responses against dietary antigens and gastrointestinal commensal flora, has been shown to be causally dependent on the colonization of certain Clostridiales that abundantly produce short-chain fatty acids $(10,11)$. Among those gut microbiota-derived short-chain fatty acids, butyrate is found to be a key factor for maintaining the integrity of CD326+ intestinal epithelial cells and mitigating graft-versus-host disease in a murine model of allogenic hematopoietic cell transplantation (4). However, homeostatic maintenance of intestinal Tregs appears to require not only the indigenous Clostridia species but flexible diversity of the host TCR repertoire. Transgenic mice genetically engineered to express a restricted TCR $\beta$ repertoire spontaneously developed severe colitis in association with hyperactivation of T helper 17 cells (Th17) and a striking decrease in a special subset of peripherally derived Tregs responsible for the recognition of intestinal microbiota (12). These "limited mice" showed no apparent alteration in the composition of commensal flora including segmented filamentous bacteria, a well-known inducer of Th17 cells in the small intestine in mice. Additionally, colonic inflammation observed in these mice is ameliorated by "total gut decontamination" by use of antibiotics cocktail, suggesting that TCR epitopes of effector Th17 cells are originated from gut microbiota rather than "self" antigens associated with autoimmunity. In this context, it is critically important to note that the use of broad-spectrum antibiotics disrupting anaerobic flora increases the risk for severe colonic graft-versus-host disease after allogeneic hematopoietic cell transplantation in human patients as well as in mice models (5). Importantly, the colon lamina propria of carbapenem antibiotic-treated mice is characterized by high local levels of IL-23 and accumulation of effector CD4+ $\mathrm{T}$ cells concomitantly with reduced colonization of Clostridiales and increased abundance of Akkermansia muciniphila, a unique bacterium that disrupts the intestinal epithelium junction by degrading luminal mucins as a source of carbohydrates and nitrogen.

More surprisingly, ongoing studies in patients with cancer highlight the crucial impact of gut microbiota on immune checkpoint immunotherapies using antibodies against programmed cell death protein 1 (PD-1) and its ligand $(7,8)$. Analysis of fecal samples from melanoma patients treated with anti-PD-1 immunotherapy revealed that the abundance of Ruminococcaceae in fecal microbiota is an indicator for good clinical responses, whereas that of Bacteroidales is a negative predictor (7). Another study including patients with advanced cancers showed that the prior use of antibiotics significantly compromised the clinical benefit of immune checkpoint inhibition, while the dominant gastrointestinal colonization of A. muciniphila was positively correlated with better responses after PD-1-based immunotherapy (8). Notably, A. municiphila has been also shown to be associated with the development of autoimmunity against the central nervous system such as multiple sclerosis $(13,14)$, suggesting a unique immunodominant role of this particular mucin-degrading anaerobic microorganism.

It is also well known that "the first microbial gut colonizers" play an essential role for the development and shaping of the early immune system in neonates and infants (15). For instance, perinatal exposure to the Bisphenol A, a chemical found in daily consumed plastics such as the coating of food and drink packages, results in reduced frequencies of Th1/Th 17 cells in the intestinal mucosa and subsequently leads to an altered glucose sensitivity, a defective IgA secretion and a fall of Bifidobacteriales in a mice model (16). The importance of "early colonizers" warrants the 
development of strategies for altering dysbiosis of infant microbiota by personalized functional nutrition.

Collectively, these observations strongly indicate that the interaction of gastrointestinal tract and indigenous microbiota is a life-long key regulator of well-balanced immunity and tolerance possibly by shaping adaptive immune cell repertoire.

\section{CURRENT RISE OF HIGH-THROUGHPUT IMMUNE REPERTOIRE SEQUENCING}

\section{Development of Massively Parallel Immunosequencing}

The first attempt to evaluate human adaptive immune repertoire by use of NGS was independently reported by three groups in 2009 (17-19). They developed a method to comprehensively and semiquantitatively determine DNA sequences of the rearranged V-D-J gene segments encoding the third complementarity-determining region (CDR3) of TRB (TCR $\beta)$ and $\operatorname{IgH}$ loci in a given lymphocyte population. Since these pivotal studies, a rapidly increasing number of researchers have installed NGS-based high-throughput sequencing of TCR (TCR-seq) and BCR/immunoglobulin (BCR/ Ig-seq) clonotypes to elucidate the characteristics and dynamics of immune repertoire in healthy individuals and patients with immune dysregulation (20-25). The introduction of this innovative approach has so far had a huge impact on basic and clinical immunologic researches and is probably beginning to change our understanding of the immune system as a whole.

The most established use of immune repertoire deep sequencing is a clinical analysis for quantitating minimal residual disease of human T-cell or B-cell neoplasms after chemotherapy or hematopoietic cell transplantation $(26,27)$. These applications have shown improved sensitivity compared with conventional assays, such as CDR3-specific PCR and multicolor flow cytometry, thus will be useful for bedside decision-making of hematologic clinicians. Moreover, BCR/Ig-seq can be used for in vivo tracing of $\mathrm{B}$ cell dynamics after vaccination and cost-effective monoclonal antibody engineering by shortcuts of labor-intensive screening procedures (28).

In its simplest form, TCR-seq and BCR/Ig-seq comprise of three essential working processes: (i) PCR amplification of V-D-J (for TRB, TRD and $I g H$ ) or V-J (for TRA, TRG, and $I g L$ ) gene segments, (ii) massively parallel sequencing of the PCR amplicons, and (iii) allignment of NGS reads by use of sophisticated bioinformatic technologies. However, many technical caveats still exist in these approaches $(29,30)$. For example, limited sampling from peripheral blood or particular tissues/organs always raises the problem of "unseen clones." In addition, the possibility of sequencing errors and amplification bias is theoretically unavoidable because it is inherent in PCR-based NGS platforms and methods for NGS library preparation. Also, the selection of the starting material, DNA or RNA, also significantly affects the quality of immune repertoire analyses. DNA-based approaches have an advantage in terms of sample preparation and storage but require complex PCRs using a multiplexed set of $\mathrm{V}$ and $\mathrm{J}$ segment-specific primers with the large reaction size because the template sequence for each TCR or BCR subunit loci exists as only single copy per cell. In contrast, RNA-based analyses, most commonly by using $5^{\prime}$-rapid amplification of cDNA ends, are capable of more comprehensive coverage and relatively unbiased amplification of the intended cDNA templates with a single pair of primers at the cost of the drawback that the read number of target amplicons is influenced by cell-to-cell variation in TCR or BCR mRNA expression levels. Finally, more serious limitation of these methodologies is a difficulty in exact pairing of variable region information ( $\alpha$ and $\beta$ units for $\alpha \beta$ TCR, $\gamma$ and $\delta$ units for $\gamma \delta \mathrm{TCR}$, and immunoglobulin heavy and light chains for BCR/Ig) that determines antigen/epitope specificity of each clonotype.

To overcome these impediments, our group has developed a novel high-throughput TCR repertoire sequencing method that combines RNA-based NGS and single-cell multiplex reverse transcriptase PCR assays for profiling TCR clonotypes with information regarding the CDR3 sequences of paired TCR $\alpha$ and TCR $\beta$ subunits (31-33). To perform relatively unbiased parallel sequencing, we installed adaptor ligation-mediated PCR for NGS library construction. With the help of this technology, we could comprehensively identify cytomegalovirus (CMV) pp65 antigenspecific paired TCR clonotypes of peripheral blood $\mathrm{T}$ cells obtained from HLA-A ${ }^{\star} 02$-positive healthy individuals. We found that HLA-A ${ }^{\star} 02$-restricted CMVpp65-specific CD8+ T-cells were extremely oligoclonal and consisted of a single or a few superdominant clones. When transduced into TCR-null Jurkat cells engineered to lack endogenous TCR by CRISPR-Cas 9 system, these superdominant TCRs showed significantly higher affinities to HLA-A*02/CMVpp65 tetramers compared with other minor TCR clonotypes. Notably, such dominant TCR clonotypes were highly shared among different individuals and more enriched in stem memory $\mathrm{T}$ cells than in the central memory or effector memory $\mathrm{T}$ cell subpopulations. These observations may suggest that stem memory T-cell subset is a reservoir of highly functional and highly shared $\mathrm{T}$ cells responsible for protective immunity against chronically infected pathogens. Similarly, several studies using NGS-based TCR repertoire analysis have revealed that the sharing of TCR clonotypes among different individuals is a common phenomenon at least in rodents and humans $(20,34,35)$. It is worthy of note that the TCR repertoire of zebrafish (Danio rerio), consisted of only a few thousands TCR $\alpha$ and TCR $\beta$ clonotypes per individual, also contains such "shared" fractions (Yasuko Honjo, Hiroyuki Sato, and Tatsuo Ichinohe, unpublished observations). Intriguingly, zebrafish T cells bearing shared TCRs are reported to show predominant expansion in response to diverse antigenic stimuli, suggesting high crossreactivity of these public $\mathrm{T}$ cell clones in system-wide T-cell repertoires of teleost fish (36). Given their advantages in smaller size and similarity of immune cells compared with the other mammalian counterparts, zebrafish will provide an ideal model to investigate the whole adaptive immune repertoire in the era of NGS-based high-throughput TCR-seq and BCR/Ig-seq at the single-individual level (37-39).

\section{Paired Immune Repertoire Sequencing by Advanced Single-Cell Transcriptomics}

Currently, more sophisticated methods to identify paired immune repertoire by use of single-cell RNA sequencing have been 
developed and some of them have already become commercially available $(30,40)$. In these pairing technologies, the common platform for separating bulk cell populations into single cells is flow cytometric sorting or encapsulation in droplet emulsions. Single-cell sorting into microwell plates is usually more feasible and less expensive, while the number of cells that can be analyzed is limited by the size and number of wells in the plates (41). In contrast, recent innovation in droplet-based microfluidics has facilitated ultra-large-scale paired sequencing from millions of cells, although the efficiencies of cell encapsulation as well as higher cost per analysis are still major challenges (42). It is expected that the introduction of such high-resolution singlecell immune receptor genomics will rapidly revolutionize and deepen our understanding of the hierarchy of adaptive immune cell repertoire. More recently, computational analytical tools are developed to predict the shared core motifs of particular epitopespecific CDR3 sequences by using several thousands of singlecell-derived TCR $\alpha$ and TCR $\beta$ sequences $(43,44)$. The systematic accumulation of paired immune repertoire sequences combined with the whole single-cell transcriptome data may ultimately lead to the development of long-awaited algorithm that can predict the function and epitope specificity of a given $\mathrm{T}$ cell or B cell in the not too distant future.

\section{POTENTIAL APPLICATIONS OF HIGH- THROUGHPUT IMMUNE SEQUENCING TO DISSECT HOST-MICROBIOME IMMUNE HOMEOSTASIS}

\section{Comprehensive Analysis of Gut- Microbiota-Reactive Immune Repertoires}

Although high-throughput immune cell repertoire analysis in the context of host-microbiome interaction is an area of open research, the most promising application of this approach is comprehensive detection of gut-microbiota-reactive $\mathrm{T}$ - or B-cell clonotypes in the circulating blood or peripheral tissues of the hosts. Very importantly, by using live-cell CD154 expression assay, it has recently been shown that healthy human adults possess a substantial amount of circulating CD4+ T cell populations reactive against bacterial lysates of gastrointestinal commensals at frequencies of 40-500 per millions of total CD4+ T cells depending on each bacterial species. The majority of these gut-microbiotareactive CD4+ $\mathrm{T}$ cells had a memory phenotype with relatively high expression of mucosa homing receptors and a Th17 marker (CD161) and was further enriched in the intestinal tissues (45). Also of note is that NGS-based TCR-seq of these gut-microbiotareactive $\mathrm{CD} 4+\mathrm{T}$ cell populations revealed one to several hundred TCR $\beta$ clonotypes putatively responsible for each bacteria-specific reactivity with significant overlap (crossreactivity) against closely related species (e.g., Escherichia coli and Bifidobacterium animalis subsp.). Furthermore, these microbiota-reactive CD4+ T cells were polarized to IL17A+ single producers after treatment with inflammatory cytokine cocktails or in patients with inflammatory bowel diseases, suggesting that some of these CD4+ T cell clones function as effectors rather than bystanders at least in selected gastrointestinal inflammatory conditions (45).

Similarly, the majority of IgA- or IgG-expressing memory $\mathrm{B}$ cell repertoires residing in human terminal ileum are reported to possess antigen-specificities for the representatives of commensal flora and intestinal pathogens (46), suggesting the essential roles of gut commensal microbiota for normal and pathological BCR/Ig repertoire ontogenesis, as was extensively studied in patients with chronic human immunodeficiency virus infection (47). Further accumulation of data obtained from these types of human studies, particularly with the use of single-cell platform NGS immune repertoire analysis, as a publicly accessible database would greatly enhance our understanding of the role of gut microbiome for the ontogenesis and age/environment-associated shaping of our immune system (Figure 1).

\section{Gut Microbiota as a Possible Origin of TCR Crossreactivities}

T-cell crossreactivity is an important area of ongoing researches in system immunology and probably one of the not yet fully recognized chief principles of adaptive immune response. Consistent with this hypothesis and in contrast to the previous general belief, an elegant study using TCR $\beta$ transgenic mice proved

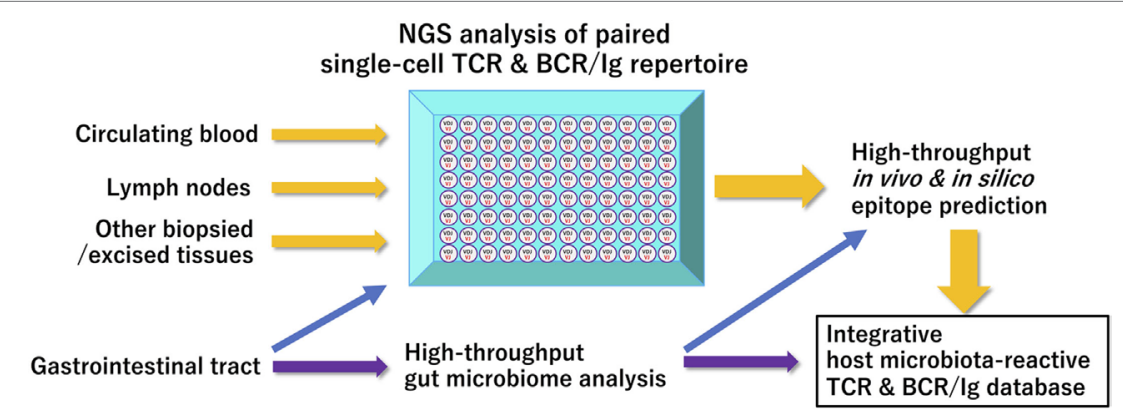

FIGURE 1 | A proposed scheme of integrated bioinformatic analysis of host-microbiome interactions Current high-throughput immune receptor sequencing technologies have facilitated comprehensive paired clonotype determination of acquired immune receptors in a given sample. Integrated accumulation of these paired immune receptor clonotypes in health and diseases with data on gut microbiome of their host origin obtained at appropriate timepoints will enhance our in-depth understanding of shared and unshared features of T- or B-cell receptor repertoire affected by specific gastrointestinal microorganisms. Abbreviations: NGS, next-generation sequencing; TCR, T-cell receptor; BCR/lgG, B-cell receptor/immunoglobulin. 
that the vast majority of negatively selected TCRs are autoreactive and endowed with crossreactivities against multiple MHC haplotypes, while crossreactive TCRs are very infrequent among preselection TCRs (48). In this context, it is very intriguing that an excellent in silico study using proteome datasets has reported the extensive sharing of possible T-cell exposed peptide motifs between human proteome and gastrointestinal microbiome (49). Very recently, $\mathrm{T}$ cell epitopes of an integrase expressed by several species of Bacteroides were shown to be a mimotope of an established pancreatic $\beta$ cell autoantigen in non-obese diabetogenic mice (50). Monocolonization studies using integrase-transgenic Bacteroides in germ-free mice demonstrated that the recruitment of diabetogenic CD8+ T cells in a microbial epitope-dependent manner. Surprisingly, these crossreactive $\mathrm{T}$ cells consistently express an invariant TCR $\alpha$ chain and function to protect the host mice from experimental colitis, suggesting that TCR repertoire of effector and regulatory $\mathrm{T}$ cells might be inherently crossreactive. Accordingly, the classical dichotomy of immunologic "self" and "non-self" should be revised and redefined because it is difficult to clearly distinguish "genetic self" and "non-genetic/microbial self” (51). NGS-based high-throughput TCR repertoire analysis will confer a more clarified overview of the origin and composition of crossreactive TCR repertoire.

\section{CONCLUSION AND PERSPECTIVES}

During the past decade, rapid innovations in genomic and bioinformatic technologies in the interdisciplinary field of microbiology and immunology have radically changed the outlook of human host-microbiome interactions and their influences on human health and disease. In particular, advanced methodologies in high-resolution adaptive immune repertoire analysis will provide an essential clue to obtain deeper understanding of the ontogeny of our immune system with its microbiome fingerprints at the individual level. Given these backgrounds in mind, it is very attractive to identify and trace the members of adaptive immune cell repertoire by single-cell TCR-seq and BCR/Ig-seq in mono- or poly-colonized germ-free animal models. Such studies will reveal the potential full diversity of TCR and BCR/Ig repertoire created by antigenic epitopes derived from intestinal commensals of particular interest, such as Clostridium and Akkermansia. In addition to well-established mice models, recently adapted gnotobiotic zebrafish husbandry may offer advantages because it can facilitate system-wide analyses at lower experimental costs, although there are still

\section{REFERENCES}

1. Castro R, Navelsaker S, Krasnov A, Du Pasquier L, Boudinot P. Describing the diversity of Ag specific receptors in vertebrates: contribution of repertoire deep sequencing. Dev Comp Immunol (2017) 75:28-37. doi:10.1016/j. dci.2017.02.018

2. Rooks MG, Garrett WS. Gut microbiota, metabolites and host immunity. Nat Rev Immunol (2016) 16:341-52. doi:10.1038/nri.2016.42

3. Honda K, Littman DR. The microbiota in adaptive immune homeostasis and disease. Nature (2016) 535:75-84. doi:10.1038/nature18848

4. Mathewson ND, Jenq R, Mathew AV, Koenigsknecht M, Hanash A, Toubai T, et al. Gut microbiome derived matabolites modulate intestinal epithelial challenges in translation of the experimental results into humans (52). Another important question is to elucidate the precise mechanism by which functional adaptive immune receptors are clonally selected through host-gut microbiome interactions and maintain long-lasting immune homeostasis. The systematic single-cell adaptive immune receptor analyses combined with large-scale microbe metagenomics in patients with various types of immunodeficiencies, autoimmune/autoinflammatory diseases, and malignant neoplasms as well as in healthy subjects with various ethnic backgrounds will provide a clue to this longstanding enigma. Future collections of such knowledge will lead to the development of effective ways for personalized immune modulation by fine tuning of the gastrointestinal homeostasis.

\section{ETHICS STATEMENT}

The studies by the authors included in this manuscript were approved by the animal experiment committee and the ethical committee of Hiroshima University.

\section{AUTHOR CONTRIBUTIONS}

TI and TM contributed equally to this work. All authors listed have made substantial contributions to text and have approved the final manuscript for submission.

\section{ACKNOWLEDGMENTS}

The authors thank Ryoko Matsumoto, Nanae Nakaju, Sachiko Fukumoto, and Masako Ninomiya for their excellent secretarial assistance. The authors would also like to thank Dr. Hiroyuki Kishi and Dr. Hiroshi Hamana (Toyama University) for their generous assistance to single-cell analyses of human TCRs in our laboratory.

\section{FUNDING}

This study was supported in part by Grants-in-Aid from the Ministry of Education, Culture, Sports, Science, and Technology of Japan (\#15K14879 to TI, \#16K19576 to TM, \#16K07175 to TK), Grants-in-Aid from the Japan Agency for Medical Research and Development (AMED) (\#17ek0510022h0001 to TI), and the Program of the network-type Joint Usage/Research Center for Radiation Disaster Medical Science of Hiroshima University, Nagasaki University, and Fukushima Medical University.

cell damage and mitigate graft-versus-host disease. Nat Immunol (2016) 17:505-13. doi:10.1038/ni.3400

5. Shono Y, Docampo MD, Peled JU, Peobelli SM, Veraldi E, Tsai JJ, et al. Increased GVHD-related mortality with broad-spectrum antibiotic use after allogeneic stem cell transplantation in human patients and mice. Sci Transl Med (2016) 8:339ra71. doi:10.1126/scitranslmed.aaf2311

6. Peled JU, Devlin SM, Staffas A, Lumish M, Khanin R, Littman ER, et al. Intestinal microbiota and relapse after hematopoietic-cell transplantation. J Clin Oncol (2017) 35:1650-9. doi:10.1200/JCO.2016.70.3348

7. Gopalakrishnan V, Spencer CN, Nezi L, Reuben A, Andrews MC, Karpinets TV, et al. Gut microbiome modulates response to anti-PD-1 immunotherapy in melanoma patients. Science (2018) 359(6371):97-103. doi:10.1126/science.aan4236 
8. Routy B, Le Chatelier E, Derosa L, Duong CPM, Alou MT, Daillère R, et al. Gut microbiome influences efficacy of PD-1-based immunotherapy against epithelial tumors. Science (2018) 359(6371):91-7. doi:10.1126/science.aan3706

9. Bene K, Varga Z, Petrov VO, Boyko N, Rajnavolgyi E. Gut microbiota species can provoke both inflammatory and tolerogenic immune reponses in human dendritic cells mediated by retinoic acid receptor alpha ligation. Front Immunol (2017) 8:427. doi:10.3389/fimmu.2017.00427

10. Atarashi K, Tanoue T, Shima T, Kuwahara T, Momose Y, Cheng G, et al. Induction of colonic regulatory $\mathrm{T}$ cells by indigenous Clostridium species. Science (2011) 331:337-41. doi:10.1126/science.1198469

11. Atarashi K, Tanoue T, Oshima K, Suda W, Nagano Y, Nishikawa H, et al. Treg induction by a rationally selected mixture of clostridia strains from the human microbiota. Nature (2013) 500:232-6. doi:10.1038/nature12331

12. Nishio J, Baba M, Atarashi K, Tanoue T, Negishi H, Yanai H, et al. Requirement of full TCR repertoire for regulatory $\mathrm{T}$ cells to maintain intestinal homeostasis. Proc Natl Acad Sci U S A (2015) 112:12770-5. doi:10.1073/pnas.1516617112

13. Jangi S, Gandhi R, Cox LM, Li N, von Glehn F, Yan R, et al. Alterations of the human gut microbiome in multiple sclerosis. Nat Commun (2016) 7:12015. doi:10.1038/ncomms12015

14. Cekanaviciute E, Yoo BB, Runia TF, Debelius JW, Singh S, Nelson CA, et al. Gut bacteria from multiple sclerosis patients modulate human $\mathrm{T}$ cells and exacerbates symptoms in mouse models. Proc Natl Acad Sci U S A (2017) 114:10713-8. doi:10.1073/pnas.1711235114

15. Milani C, Duranti S, Bottacini F, Casey E, Turroni F, Mahony J, et al. The first microbial colonization of the human gut: composition, activities, and health implications of the infant gut microbiota. Microbiol Mol Biol Rev (2017) 81:e36-17. doi:10.1128/MMBR.00036-17

16. Malaisè T, Menard S, Cartier C, Galutier E, Laserre F, Harkar C, et al. Gut dysbiosis and impairment of immune homeostasis in perinatally-exposed mice to bisphenol A precede obese phenotype development. Sci Rep (2017) 7:14472. doi:10.1038/s41598-017-15196-w

17. Robins HS, Campregher PV, Srivastava SK, Wacher A, Turtle CJ, Kahsai O, et al. Comprehensive assessment of T-cell receptor $\beta$-chain diversity in $\alpha \beta$ T cells. Blood (2009) 114:4099-107. doi:10.1182/blood-2009-04-217604

18. Freeman JD, Wallen RL, Webb JR, Nelson BH, Holt RA. Profiling of the T-cell receptor beta-chain repertoire by massive parallel sequencing. Genome Res (2009) 19:1817-24. doi:10.1101/gr.115428.110

19. Boyd SD, Marshall EL, Merker JD, Maniar JM, Zhang LN, Sahaf B, et al. Measurement and clinical monitoring of human lymphocyte clonality by massively parallel VDJ pyrosequencing. Sci Transl Med (2009) 1:12ra23. doi:10.1126/scitranslmed.3000540

20. Robins HS, Srivastava SK, Campregher PV, Turtle CJ, Andriesen J, Riddell SR, et al. Overlap and effective size of the human CD8+ T cell receptor repertoire. Sci Transl Med (2010) 2:47ra46. doi:10.1126/scitranslmed.3001442

21. Wang C, Sanders CM, Yang Q, Schroeder HW Jr, Wang E, Babradeh F, et al. High throughput sequencing reveals a complex pattern of dynamic interrelationships among human T cell subsets. Proc Natl Acad Sci U S A (2010) 107:1518-23. doi:10.1073/pnas.0913939107

22. von Büdingen H-C, Kuo TC, Sirota M, van Belle CJ, Apeltsin L, Granville J, et al. B cell exchange across the blood-brain barrier in multiple sclerosis. J Clin Invest (2012) 122:4533-44. doi:10.1172/JCI63842

23. vanHeijstJW,CeberioI,LipumaMB,SamilioDW, WasilewskiGD, GonzalesAM, et al. Quantitative assessment of $\mathrm{T}$ cell repertoire recovery after hematopoietic stem cell transplantation. Nat Med (2013) 19:372-7. doi:10.1038/nm.3100

24. Putintseva EV, Britanova OV, Staroverov DB, Merzlyak EM, Turchaninova MA, Shugay M, et al. Mother and child $\mathrm{T}$ cell receptor repertoires: deep profiling study. Front Immunol (2013) 4:463. doi:10.3389/fiimu.2013.00463

25. Zvyagain IV, Pogorelyy MV, Ivanova ME, Komech EA, Shugay M, Bolotin DA, et al. Distinctive properties of identical twin's TCR repertoires revealed by high-throughput sequencing. Proc Natl Acad Sci U S A (2014) 111:5980-5. doi:10.1073/pnas.1319389111

26. Weng WK, Armstrong R, Arai S, Desmarais C, Hoppe R, Kim YH. Minimal residual disease monitoring with high-throughput sequencing of $\mathrm{T}$ cell receptors in cutaneous T cell lymphoma. Sci Transl Med (2013) 5:214ra171. doi:10.1126/scitranslmed.3007420

27. Langerank AW, Bruggemann M, Davi F, Darzentas N, van Dongen JJM, Gonzalez D, et al. High-throughput immunogenetics for clinical research applications in immunohematology: potential and challenges. JImmunol (2017) 198:3765-74. doi:10.4049/jimmunol.1602050
28. Boyd SD, Crowe JE Jr. Deep sequencing and human antibody repertoire analysis. Curr Opin Immunol (2016) 40:103-9. doi:10.1016/j.coi.2016.03.008

29. Woodsworth DJ, Catellarin M, Holt RA. Sequence analysis of T-cell repertoires in health and disease. Genome Med (2013) 5:98. doi:10.1186/gm502

30. Friedensohn S, Khan TA, Reddy ST. Advanced methodologies in highthroughput sequencing of immune repertoires. Trends Biotechnol (2017) 35:203-14. doi:10.1016/j.tibtech.2016.09.010

31. Kitaura K, Shin-I T, Matsutani T, Suzuki R. A new high-throughput sequencing method for determining diversity and similarity of T cell receptor (TCR) $\alpha$ and $\beta$ repertoires and identifying potential new invariant TCR $\alpha$ chains. BMC Immunol (2016) 17:38. doi:10.1186/s12865-016-0177-5

32. Miyama T, Kawase T, Kitaura K, Chishaki R, Shibata M, Oshima K, et al. Highly functional T-cell receptor repertoires are abundant in stem memory $\mathrm{T}$ cells and highly shared among individuals. Sci Rep (2017) 7:3663. doi:10.1038/ s41598-017-03855-x

33. Kobayashi E, Mizukoshi E, Kishi H, Ozawa T, Hamana H, Nagai T, et al. A new cloning and expression system yields and validates TCRs from blood lymphocytes of patients with cancer within 10 days. Nat Med (2013) 19: 1542-6. doi:10.1038/nm.3358

34. Venturi V, Quingley M, Greenaway HY, Ng PC, Ende ZS, McIntosh T, et al. A mechanism for TCR sharing between T cell subsets and individuals revealed by pyrosequencing. J Immunol (2011) 186:4285-94. doi:10.4049/ jiimunol.1003898

35. Li H, Ye C, Ji G, Wu X, Xiang Z, Li Y, et al. Recombinatorial biases and convergent recombination determine interindividual TCR $\beta$ sharing in murine thymocytes. J Immunol (2012) 189:2404-13. doi:10.4049/jiimunol.1102087

36. Covacu R, Philip H, Jaronen M, Almeida J, Kenison J, Darko S, et al. Systemwide analysis of the T-cell response. Cell Rep (2016) 14:2733-44. doi:10.1016/j. celrep.2016.02.056

37. Weinstein JA, Jiang N, White RA III, Fisher DS, Quake SR. High-throughput sequencing of the zebrafish antibody repertoire. Science (2009) 324:807-10. doi:10.1126/science. 1170020

38. Fillatreau S, Six A, Magadan S, Castro R, Sunyer JO, Boudinot P. The astonishing diversity of Ig classes and B cell repertoires in teleost fish. Front Immunol (2013) 4:28. doi:10.3389/fimmu.2013.00028

39. Iwanami N, Hess I, Schorpp M, Boehm T. Studying the adaptive immune system in zebrafish by transplantation of hematopoietic precursor cells. Methods Cell Biol (2017) 138:151-61. doi:10.1016/bs.mcb.2016.08.003

40. Stubbington MJT, Rozenblatt-Rosen O, Regev A, Teichman SA. Single-cell transcriptomics to explore the immune system in health and disease. Science (2017) 358:58-63. doi:10.1126/science.aan6828

41. Howie B, Sherwood AM, Berkebile AD, Berka J, Emerson RO, Williamson DW, et al. High-throughput pairing of T cell receptor $\alpha$ and $\beta$ sequences. Sci Transl Med (2015) 7:301ra131. doi:10.1126/scitranslmed.aac5624

42. McDanile JR, DeKosky BJ, Tanno H, Ellington AD, Georgiou G. Ultra-highthroughput sequencing of the immune receptor repertoire from millions of lymphocytes. Nat Protoc (2016) 11:429-42. doi:10.1038/nprot.2016.024

43. Dash P, Fiore-Gartland AJ, Hertz T, Wang GC, Sharma S, Souquette A, et al. Quantifiable predictive features define epitope-specific $\mathrm{T}$ cell receptor repertoires. Nature (2017) 547:89-93. doi:10.1038/nature22383

44. Glanville J, Huang H, Nau A, Hatton O, Wager LE, Rubelt F, et al. Identifying specifity groups in the T cell receptor repertoire. Nature (2017) 547:94-8. doi:10.1038/nature22976

45. Hegazy AN, West NR, Stubbington MJT, Wendt E, Suijker KIM, Datsi A, et al. Circulating and tissue-resident CD4+ T cells with reactivity to intestinal microbiota are abundant in healthy individuals and function is altered during inflammation. Gastroenterology (2017) 153:1320-37. doi:10.1053/j. gastro.2017.07.047

46. Benckert J, Schmolka N, Kreschel C, Zoller MJ, Sturm A, Wiedenmann B, et al. Tha majority of intestinal IgA+ and IgG+ plasmablasts in the human gut are antigen-specific. J Clin Invest (2011) 121:1946-55. doi:10.1171/JCI44447

47. Nguyen QN, Himes JE, Martinez DR, Permar SR. The impact of the gut microbiota on humoral immunity to pathogens and vaccination in early infancy. PLoS Pathog (2016) 12:e1005997. doi:10.1371/journal.ppat.1005997

48. McDonald BJ, Bunker JJ, Erickson SA, Oh-Hora M, Bendelac A. Crossreactive $\alpha \beta \mathrm{T}$ cell receptors are the predominant targets of thymocyte negative selection. Immunity (2015) 43:859-69. doi:10.1016/j.immuni.2015.09.009

49. Bremel RD, Homan EJ. Extensive T-cell epitope repertoire sharing among human proteome, gastrointestinal microbiome, and pathogenic bacteria: 
implications for the definition of self. Front Immunol (2015) 6:538. doi:10.3389/ fiimu. 2016.00538

50. Nanjundappa RH, Ronchi F, Wang J, Clemente-Casares X, Yamanouchi J, Sokke Umeshappa C, et al. A gut microbial mimic that hijacks diabetogenic autoreactivity to suppress colitis. Cell (2017) 171:655-67. doi:10.1016/j. cell.2017.09.022

51. Root-Bernstein R. Autoimmunity and the microbiome: T-cell receptor mimicry of "self" and microbial antigens mediates self tolerance in holobionts. Bioessays (2016) 38:1068-83. doi:10.1002/bies.201600083

52. Melancon E, Gomez De La Torre Canny S, Sichel S, Kelly M, Wiles TJ, Rawls JF, et al. Best practices for germ-free derivation and gnotobiotic zebrafish husbandry. Methods Cell Biol (2017) 138:61-100. doi:10.1016/bs.mcb.2016.11.005
Conflict of Interest Statement: RS is the chief executive director of Repertoire Genesis Incorporation. KK and HS are employees of Repertoire Genesis Incorporation. TS is the chief executive director of BITS Co. Ltd. No other authors have relevant conflicts of interest to declare.

Copyright (๑) 2018 Ichinohe, Miyama, Kawase, Honjo, Kitaura, Sato, Shin-I and Suzuki. This is an open-access article distributed under the terms of the Creative Commons Attribution License (CC BY). The use, distribution or reproduction in other forums is permitted, provided the original author(s) and the copyright owner are credited and that the original publication in this journal is cited, in accordance with accepted academic practice. No use, distribution or reproduction is permitted which does not comply with these terms. 